\title{
Population synthesis modelling of luminous infrared galaxies at intermediate redshift
}

\author{
E. Giovannoli ${ }^{1}$, V. Buat ${ }^{1}$, S. Noll ${ }^{2}$, D. Burgarella ${ }^{1}$, and B. Magnelli ${ }^{3}$ \\ ${ }^{1}$ Laboratoire d'Astrophysique de Marseille, OAMP, Université Aix-Marseille, CNRS, 38 rue Frédéric Joliot-Curie, \\ 13388 Marseille Cedex 13, France \\ e-mail: [elodie.giovannoli, veronique.buat, denis.burgarella]@oamp.fr \\ 2 Institut für Astro- und Teilchenphysik, Universität Innsbruck, Technikerstr. 25/8, 6020 Innsbruck, Austria \\ e-mail: Stefan.Noll@uibk.ac.at \\ 3 Max-Planck-Institut für Extraterrestrische Physik (MPE), Postfach 1312, 85741 Garching, Germany \\ e-mail: magnelli@mpe.mpg.de
}

Received 30 April 2010 / Accepted 3 June 2010

\begin{abstract}
Context. Luminous InfraRed Galaxies (LIRGs) are particularly important for studying the build-up of the stellar mass from $z=1$ to $z=0$, and for determining physical properties of these objects at redshift 0.7 . LIRGs are now identified as playing a major role in galaxy evolution from $z=1$ to 0 . The global star formation rate (SFR) at $z \sim 0.7$ is mainly produced by LIRGs.

Aims. We perform a multiwavelength study of an LIRGs sample in the Extended Chandra Deep Field South at $z=0.7$, selected at $24 \mu \mathrm{m}$ by MIPS onboard Spitzer Space Telescope and detected in 17 filters. Data go from the near-ultraviolet to the mid-infrared. This multiwavelength dataset allows us to place strong constraints on the spectral energy distributions (SEDs) of galaxies, and thus to efficiently derive physical parameters such as the SFR, the total infrared luminosity, attenuation parameters, and star formation history. We distinguish a subsample of galaxies detected at $70 \mu \mathrm{m}$, which we compare to the rest of the sample to investigate the relative importance of this wavelength in determining of the physical parameters. An important part of this work is elaboration of a mock catalogue that allows us to have a reliability criterion for the derived parameters.

Methods. We studied LIRGs by means of the SED-fitting code CIGALE. At first, this code creates synthetic spectra from the Maraston stellar population models. The stellar population spectra are attenuated by using a synthetic Calzetti-based attenuation law before adding the dust emission as given by the infrared SED library. The originality of CIGALE is that it allows us to perform consistent fits of the dust-affected ultaviolet-to-infrared wavelength range. This technique appears to be a very powerful tool in the case where we can have access to a dataset that is well-sampled over a wide range of wavelengths.

Results. We are able to derive a star formation history and to estimate the fraction of infrared luminosity reprocessed by an active galactic nucleus. We study the dust temperatures of our galaxies detected at $70 \mu \mathrm{m}$ and find them colder than predicted by models. We also study the relation between the SFR and the stellar mass and do not find a tight correlation between either of them, but instead a flat distribution and a large scatter, which is interpreted in terms of variations in star formation history.
\end{abstract}

Key words. galaxies: evolution - galaxies: formation - galaxies: fundamental parameters - galaxies: stellar content

\section{Introduction}

Luminous InfraRed Galaxies (LIRGs) are commonly defined as galaxies whose infrared (IR, 8-1000 $\mu \mathrm{m}$ ) emission is higher than $10^{11} L_{\odot}$ and lower than $10^{12} L_{\odot}$. In a pioneering work Chary \& Elbaz (2001, hereafter CE01), identified them as the main contributor to the cosmic IR background detected by ISO (Infrared Space Observatory); the deep Spitzer/MIPS (Rieke et al. 2004) surveys have confirmed that they play a major role in galaxy evolution at intermediate redshift $(z=0.5-1)$. Indeed the IR luminosity function is found to evolve with $z$ and to shift towards higher luminosities when $z$ increases (Le Floc'h et al. 2005), (Pérez-González et al. 2005); as a consequence, LIRGs are rare objects in the nearby universe but become the major contributors to the star formation density measured in IR at $z=$ 0.5-1 (Le Floc'h et al. 2005; Caputi et al. 2007; Magnelli et al. 2009). The evolution of LIRGs as a function of $z$ concerns not only their contribution to the luminosity density but also their physical characteristics. Most of the LIRGs populating the local universe are located in interacting violently systems (Ishida 2004). Conversely, at $z \sim 1$ only $30 \%$ of LIRGs exhibit features linked to violent merging (Bell et al. 2007; Zheng et al. 2007): most of them look like bright spirals that experience a secular evolution without violent events. This morphological difference between local and distant LIRGs is corroborated by the analysis of their star formation rate (SFR). Whereas local LIRGs are experiencing a strong starburst, distant LIRGs do not seem to strongly depart from the mean SFR - stellar mass (hereafter $M_{\star}$ ) relation found at $z=1$ (Elbaz et al. 2007).

Quite naturally, most of the studies devoted to LIRGs are only based on IR data. Recently several teams have aimed at studying local LIRGs by combining a large set of wavelengths and observations (Burgarella et al. 2005; Kaviraj et al. 2008; Da Cunha 2008). Such studies are scarcer at higher $z$. Zheng et al. (2007) have combined ultraviolet (UV), optical, and IR observations and built mean spectral energy distribution (SEDs) 
of IR selected galaxies at $z=0.7$. They are found to be similar to those of nearby normal galaxies with lower IR luminosities (hereafter $L_{\text {dust }}, L(8-1000 \mu \mathrm{m})$ ).

The availability of large datasets of photometric data have led to a renewal of stellar population synthesis codes assuming more or less complex star formation histories (SFHs). The combination of galaxy simulations with realistic SED modelling improves our understanding of the uncertainties of these fitting methods to estimate SFRs, $M_{\star}$ or population ages (Wuyts et al. 2009; Conroy et al. 2009a; Lee et al. 2009; Dye 2008). Most of these studies only deal with the stellar UV-near-infrared (NIR) SEDs of galaxies. Since LIRGs emit most of their energy in IR we must account for the entire UV-to-IR SEDs to characterise them. Up to now there have been few attempts to combine stellar and dust emissions in the modelling of galaxy SEDs. Stars and dust interact in a very complex way in galaxies, and a detailed description of this interplay is not always consistent with an SED-fitting code aimed at studying large samples of galaxies (Dopita et al. 2005). The GRASIL (GRAphite and SILicate) code (Silva et al. 1998; Panuzzo et al. 2005) produces UV-toIR SEDs based on a set of physical processes; (Iglesias-Páramo et al. 2007) used GRASIL to generate a synthetic library of galaxy SEDs and fit a multiwavelength dataset of UV selected galaxies from $z=0.2$ to 0.7. Da Cunha et al. (2008) developed a physically motivated model by combining three IR components to the stellar populations synthesis code of Bruzual \& Charlot (2003). Burgarella et al. (2005) and Noll et al. (2009b) followed a similar approach and propose a code that calculates the effect of dust on galaxy SEDs in a consistent way, and the code is versatile enough to be used at different redshifts and with different SFHs. All these codes that combine stellar and dust populations are very efficient at constraining dust attenuation on the basis of an energetic budget: the IR dust emission originates from the stellar light absorbed in the UV-NIR part of the spectrum. In this paper we apply a multiwavelength analysis from the far-ultraviolet (FUV) to the IR, based on SED-fitting, on a sample of $z=0.7$ LIRGs selected at $24 \mu \mathrm{m}$. Our analysis is based on the code CIGALE (Code Investigating GALaxy Emission $^{1}$ ) (Noll et al. 2009b). It is the first application of this code to intermediate redshift galaxies mostly emitting in IR. Our aim is to study this galaxy sample, representative of LIRGs at intermediate redshift, in a very homogeneous and systematic way to determine the main characteristics of their stellar populations and dust emission. We will re-visit dependence of the dust temperature (deduced from the IR SED) on $L_{\text {dust }}$ and the SFH of these galaxies.

In Sect. 2 we describe the sample and the multiwavelength dataset. In Sect. 3 we summarise the main characteristics of the CIGALE code and its application to our sample, in particular we build a mock catalogue to test the code performances. The results of the SED-fitting to the LIRGs sample are presented in Sect. 4. Section 5 is devoted to a discussion of these results in the framework of the temperature-luminosity relation and the specific SFRs of LIRGs at $z=0.7$, which is compared to model predictions.

We adopt an $H_{0}=70 \mathrm{~km} \mathrm{~s}^{-1} \mathrm{Mpc}^{-1}, \Omega_{\mathrm{M}}=0.3, \Omega_{\Lambda}=0.7$ cosmology throughout this paper.

\section{The sample and data}

The Chandra Deep Field South (CDFS, Giacconi et al. 2002) is one of the most intensely studied regions of the sky, with

\footnotetext{
${ }_{1}$ Web address to use CIGALE: http://www . oamp.fr/cigale/
}

observations stretching from the X-ray to the radio, including UV, optical, IR, and submillimetre imaging from space, as well as from the largest terrestrial observatories. The region of the CDFS ( $\left.\alpha=3^{\mathrm{h}} 32^{\mathrm{m}} 00^{\mathrm{s}}, \delta=-27^{\circ} 35^{\prime} 00^{\prime \prime}, \mathrm{J} 2000.0\right)$ was observed with the MIPS instrument onboard the Spitzer Space Telescope (Werner et al. 2004) in 2004 January as part of the MIPS Guaranteed Time Observing programme.

Le Floc'h et al. (2005) used the 2955 sources detected at $24 \mu \mathrm{m}$ by MIPS with flux at $24 \mu \mathrm{m}>83 \mu \mathrm{Jy}$ at the $80 \%$ completeness limit (Papovich et al. 2004) to build the IR luminosity function from $z=1$ to 0 . Photometric redshifts from COMBO17 (Classifying Objects by Medium-Band Observations in 17 filters, Wolf et al. 2004) were used for sources at $z \leqslant 1.2$ (see Le Floc'h et al. 2005, for more details). Buat et al. (2007a) selected a subsample of 190 LIRGs at $0.6<z<0.8$ and measured the near ultraviolet (NUV) emission of these galaxies on deep Galaxy Evolution Explorer (GALEX) images. Eighty percent of the 190 LIRGs were detected at NUV. The four IRAC bands $(3.6 \mu \mathrm{m}, 4.5 \mu \mathrm{m}, 5.8 \mu \mathrm{m}$, and $8.0 \mu \mathrm{m})$ are also available for these sources (N. Bavouzet, private communication). The optical-toNIR photometry was taken from a K-selected catalogue of the ECDFS( Extended CDFS, $\alpha=3^{\mathrm{h}} 32^{\mathrm{m}} 00^{\mathrm{s}}, \delta=-27^{\circ} 48^{\prime} 00^{\prime \prime}$ ) as part of the multiwavelength Survey by Yale-Chile (MUSYC, Taylor et al. $2009^{2}$ ). This catalogue consists of photometry derived from $U, U 38, B, V, R, I, z^{\prime}, J, H$, and $K$, imaging for approximately $80 \%$ of the field. The $5 \sigma$ flux limit for point-sources is $K_{\text {tot }}^{(\mathrm{AB})}=22.0$.

We cross-correlated sources selected by Buat et al. (2007a) and detected at $24 \mu \mathrm{m}$ with MUSYC data using a tolerance radius of $2^{\prime \prime}$. When a double match was found, we selected the closest object. We ignored the case where three or more optical sources can be associated with a given $24 \mu \mathrm{m}$ detection and thus eliminated 9 objects from the 190 sources. At the end we were left with 181 galaxies whose 150 have an NUV detection (see Table 1) with a mean redshift of $0.70+/-0.05$. For the 31 objects not detected in NUV, a mean redshift of $0.72+/-0.05$ was found.

The ECDFS was observed with the MIPS instrument as part of the FIDEL (Far-Infrared Deep Extragalactic Legacy Survey, PI: Dickinson) legacy programme. For the $70 \mu \mathrm{m}$ data, we used the catalogue from Magnelli et al. (2009). The detection limit for $70 \mu \mathrm{m}$ sources in this field is $3.5 \mathrm{mJy}(5 \sigma)$. We refer to this paper for more details about the source extraction, photometry, and completeness of the catalogue. We cross-correlated our 181 objects with FIDEL data thanks to their IRAC (Fazio et al. 2004) positions, with a tolerance radius of 2 ". Finally 62 galaxies were detected at $70 \mu \mathrm{m}$ (see Table 1), with a mean redshift of $0.68+/-0.04$.

The fraction of MIPS sources showing evidence of an active galactic nucleus (AGN) in their optical counterparts is less than $\sim 15 \%$ according to the VIMOS (VLT Deep Survey) and COMBO17 classifications. Synthetic models connecting the $\mathrm{X}$-ray and IR SED of AGN indicate that the emission arising from pure AGNs should be negligible (i.e., $\$ 10 \%$ ) in highredshift sources detected at $24 \mu \mathrm{m}$ (Silva et al. 2004). To have more precision about the possible fraction of AGN in our sample, we use a diagnostics colour-colour given by Stern et al. (2005) and the diagnostics of the mid-IR (MIR) slope of Brand et al. (2006) (see Sect. 3.3); we also use a specific output of the code CIGALE (see Sect. 4.2). These studies are described later in this paper.

${ }^{2}$ MUSYC collaboration: http://www . astro . yale.edu/MUSYC/ 
Table 1. The observed data: wavelengths corresponding to the filters' identification of instrument and survey.

\begin{tabular}{|c|c|c|c|}
\hline Filters & Wavelength & Instrument-Survey & Galaxies detected \\
\hline \multicolumn{4}{|l|}{ Mid-IR, IR } \\
\hline MIPS 1 & $24 \mu \mathrm{m}$ & MIPS/Spitzer - FIDEL & 181 \\
\hline MIPS 2 & $70 \mu \mathrm{m}$ & $\eta$ & 62 \\
\hline \multicolumn{4}{|l|}{ Near-IR } \\
\hline IRAC 1 & $3.6 \mu \mathrm{m}$ & IRAC-GTO Spitzer & 177 \\
\hline IRAC 2 & $4.5 \mu \mathrm{m}$ & $\|$ & 161 \\
\hline IRAC 3 & $5.8 \mu \mathrm{m}$ & " & 177 \\
\hline IRAC 4 & $8.0 \mu \mathrm{m}$ & $"$ & 161 \\
\hline \multicolumn{4}{|l|}{ UV - Optical } \\
\hline$U$ & $0.35 \mu \mathrm{m}$ & MUSYC & 179 \\
\hline U38 & $0.36 \mu \mathrm{m}$ & $"$ & 180 \\
\hline$B$ & $0.46 \mu \mathrm{m}$ & $"$ & 181 \\
\hline$V$ & $0.54 \mu \mathrm{m}$ & $"$ & 181 \\
\hline$R$ & $0.65 \mu \mathrm{m}$ & $"$ & 181 \\
\hline$I$ & $0.87 \mu \mathrm{m}$ & $"$ & 181 \\
\hline$z$ & $0.90 \mu \mathrm{m}$ & $"$ & 181 \\
\hline$J$ & $1.2 \mu \mathrm{m}$ & ” & 180 \\
\hline$H$ & $1.6 \mu \mathrm{m}$ & $”$ & 155 \\
\hline$K$ & $2.1 \mu \mathrm{m}$ & $"$ & 181 \\
\hline \multicolumn{4}{|l|}{$\overline{\mathbf{U V}}$} \\
\hline NUV & $2310 \AA$ & GALEX-DIS & 150 \\
\hline
\end{tabular}

Notes. Our sample contains 181 galaxies, of which 62 are detected at $70 \mu \mathrm{m}$.

We decided to distinguish our SED-fitting analysis between the subsample detected at $70 \mu \mathrm{m}$ and the whole sample. Hereafter we use "70 $\mu \mathrm{m}$ sample" for galaxies detected at $70 \mu \mathrm{m}$ and "total sample" for the whole set of galaxies.

\section{SED-fitting techniques: description of the code CIGALE}

The best way to derive physical parameters like the SFH is to fit the observed SED with models from a stellar population synthesis code. We use the code CIGALE which provides physical informations about galaxies by fitting their UV-to-IR SED. A first version of the code was developed by Burgarella et al. (2005) to reproduce the dust-attenuated stellar emission from the FUV to the NIR. Since $L_{\text {dust }}$ could only be provided as an input parameter, Noll et al. (2009b) especially extended the code to the far-IR (FIR) by considering dust emission models to allow a consistent treatment of dust effects on galaxy SEDs. A Bayesianlike analysis is used to derive galaxy properties, and Noll et al. (2009b) applied it to local galaxies. This work represents the first attempt to apply CIGALE to galaxies at intermediate redshift. CIGALE is based on the use of a UV-optical stellar SED plus a dust, IR-emitting component. First, UV-to-IR models are built, and second, these models are fit to the observed SEDs. The code practically fits the observed data in the UV, optical, and NIR with models generated with a stellar populations synthesis code, assuming an SFH and a dust attenuation scenario as input. The energetic balance between dust-enshrouded stellar emission and re-emission in the IR is carefully conserved by combining the UV/optical and IR SEDs. The IR SEDs are built from the Dale \& Helou (2002) templates (hereafter DH02). We refer the reader to Noll et al. (2009b) for more details.

In the following we describe parameters crucial to this study. The input parameters values are listed in Table 2.

\subsection{Stellar populations and star formation histories}

In its current version CIGALE provides two stellar population synthesis models: Maraston et al. (2005, hereafter M05) and PEGASE (Fioc \& Rocca-Volmerange 1997). Both include a treatment of thermally pulsating asymptotic-giant branch (TP-AGB) stars but in a different way. For instance, in M05 models young stellar populations are modelled with the Geneva stellar evolutionary tracks, while the Padova tracks are used in PEGASE.

TP-AGBs are particularly important for a reliable $M_{\star}$ determination at high $z$ (M05). The difference between PEGASE and M05 models lies in the weight given to the TP-AGBs. Their contribution will be systematically higher in M05 models. Models that do not consider enough TP-AGB need a massive contribution of old stars to match the observed NIR fluxes and thus overestimate $M_{\star}(\mathrm{M} 05)$. In a recent work, Tonini et al. (2009) finds that the use of PEGASE does not reproduce IRAC luminosities for nearly passive and star-forming galaxies around $z \sim 2$. For these reasons we decided to use the M05 models.

Two stellar components have been found necessary to reconstruct more accurate SFRs in actively star-forming galaxies (e.g. Erb et al. 2006; Lee et al. 2009). CIGALE uses the single stellar populations of M05 and combines two stellar populations to reproduce an old and a young stellar population. M05 models assume SFH with exponentially decreasing SFR: $\tau_{1}$ and $\tau_{2}$ represent the $e$-folding time of the old and young stellar populations, respectively. The SFR is expressed as

$$
\begin{aligned}
& \operatorname{SFR}_{1}(t)=S F R_{0,1} \cdot \mathrm{e}^{\frac{-\left(t-t_{1}\right)}{\tau_{1}}} \\
& \operatorname{SFR}_{2}(t)=S F R_{0,2} \cdot \mathrm{e}^{\frac{-\left(t-t_{2}\right)}{\tau_{2}}},
\end{aligned}
$$

where $t_{1}$ and $t_{2}$ represent the age of the old and the young stellar populations and $S F R_{0,1}$ and $S F R_{0,2}$ are the values of the $S F R$ at $t=t_{1}$ and $t=t_{2}$, respectively. 
Table 2. List of the input parameters of the code CIGALE and their selected range for a specific run.

\begin{tabular}{|c|c|c|}
\hline Parameters & Symbol & Range \\
\hline Attenuation & & \\
\hline$V$-band attenuation & $A_{V}$ & $\begin{array}{l}0.15 ; 0.30 ; 0.45 ; 0.60 ; 0.75 ; 0.90 ; 1.05 ; 1.20 ; 1.35 \\
1.5 ; 1.65 ; 1.8 ; 1.95 ; 2.1\end{array}$ \\
\hline Reduction of $A_{V}$ basic for old SP model & $f_{V}$ & $0.0 ; 0.25 ; 0.50 ; 0.75 ; 1.0$ \\
\hline \multicolumn{3}{|l|}{ IR SED } \\
\hline IR power-law slope & $\alpha$ & $1.0 ; 1.25 ; 1.5 ; 1.75 ; 2.0 ; 2.25 ; 2.5$ \\
\hline AGN-related fraction of $L_{\text {dust }}$, & $f_{\mathrm{AGN}}$ & $0.0 ; 0.001 ; 0.005 ; 0.01 ; 0.05 ; 0.1 ; 0.5 ; 0.7 ; 0.999$ \\
\hline $\begin{array}{l}\text { Star Formation History } \\
\text { metallicities (solar metallicity) }\end{array}$ & $Z$ & 0.02 \\
\hline$\tau$ of old stellar population models in $\mathrm{Gyr}$ & $\tau_{1}$ & $0.1 ; 3.0 ; 10.0$ \\
\hline ages of old stellar population models in Gyr & $t_{1}$ & 7 \\
\hline$\tau$ of young stellar population models in Gyr & $\tau_{2}$ & 20.0 \\
\hline ages of young stellar population models in Gyr & $t_{2}$ & $\begin{array}{l}0.025 ; 0.05 ; 0.075 ; 0.1 ; 0.2 ; 0.3 ; 0.4 ; 0.5 ; 0.6 ; 0.7 ; \\
0.8 ; 0.9 ; 1.0 ; 1.5 ; 2.0\end{array}$ \\
\hline fraction of young stellar population & $f_{\mathrm{ySP}}$ & $\begin{array}{l}0.0 ; 0.001 ; 0.005 ; 0.01 ; 0.05 ; 0.06 ; 0.07 ; 0.08 ; 0.09 \\
0.1 ; 0.2 ; 0.3 ; 0.4 ; 0.5 ; 0.6 ; 0.7 ; 0.8 ; 0.999\end{array}$ \\
\hline IMF & $\mathrm{S}$ & Salpeter \\
\hline
\end{tabular}

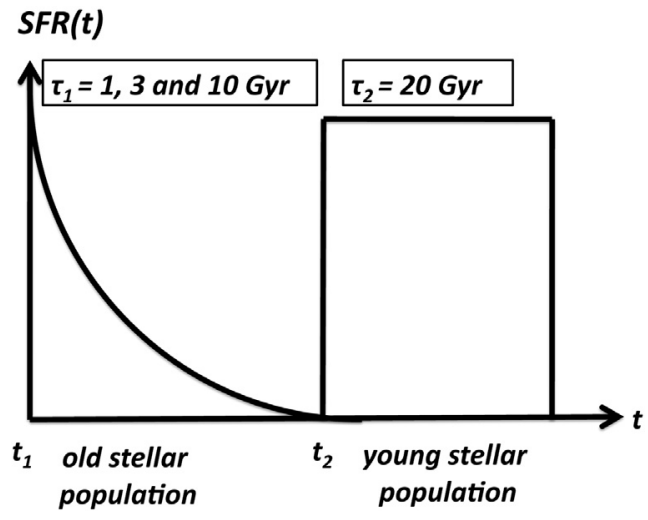

Fig. 1. Scenario of SFH: two bursts, one for the old stellar population having a moderate $e$-folding time and one for the young stellar population constant over time.

We study a sample of LIRGs lying at $z=0.7$. At this redshift, the universe was 7 Gyr old with our cosmology, so it makes no sense to consider a range up to more than 7 Gyr for the ages of both stellar populations. After several tests we realised that we cannot make a precise estimate of the $e$-folding time of the young stellar population, so we only consider an SFR constant over time. We decided to fix the age of the old stellar population $t_{1}=7 \mathrm{Gyr}$ and we suppose 3 possibilities for $\tau_{1}: \tau_{1}=1$, 3 , or 10 Gyr. We chose $t_{2}$ in the range 0.025 to 2 Gyr and considered $\tau_{2}=20 \mathrm{Gyr}$, which corresponds to a burst constant over this timescale.

The two SFH components are linked by their mass fraction. The fraction of the young stellar population (hereafter $f_{\mathrm{ySP}}$ ) corresponds to the fraction of the young stellar population mass over the total mass and thus ranges between 0 and 1 . We chose values of $f_{\mathrm{ySP}}$ in this interval to allow the choice of the bestfitting value, as we do not have any prescription for this parameter. CIGALE gives an instantaneous value of $\log _{10} S F R$ as an output, defined as $\log \left[\left(1-f_{\mathrm{ySP}}\right) * S F R_{1}+f_{\mathrm{ySP}} * S F R_{2}\right]$. In the following, the SFR will refer to this formula.

\subsection{Dust attenuation and infrared emission}

To model the attenuation by dust, the code CIGALE uses the attenuation law of Calzetti et al. (2000) as a baseline, and offers the possibility of varying the attenuation law and adding a bump. We decided to reproduce a pure Calzetti law and to add no bump. The stellar population models have to be attenuated before the IR emission can be added, depending on the dust-absorbed luminosity. The code allows us to consider that young and old stars are not affected by dust in the same way. Dust attenuation in the $V$ band, $A_{V}$, and the dust reduction factor $f_{V}$ are used for the young and the old stellar populations, respectively. The stellar light heats the dust, which emits the energy again in MIR and FIR. At these wavelengths we have few available data to reconstruct the SED. To fit IR observations CIGALE uses semiempirical one-parameter models of DH02 composed of 64 templates. These models are parametrised by $\alpha$, the power-law slope of the dust mass over heating intensity, defined as

$\mathrm{d} M_{\mathrm{d}}(U)=U^{\alpha} \mathrm{d} U$,

where $M_{\mathrm{d}}(U)$ is the dust mass heated by a radiation field at intensity $U$ and $\alpha$ is directly related to $f_{60} \mu \mathrm{m} / f_{100 \mu} \mu \mathrm{m}$ ratio flux, where $f_{60 \mu \mathrm{m}}$ and $f_{100 \mu \mathrm{m}}$ represent fluxes of the SED at 60 and $100 \mu \mathrm{m}$, respectively.

DH02 provide SEDs corresponding to different exponents $\alpha$, which is a free parameter only for the $70 \mu \mathrm{m}$ sample. The total sample only has $24 \mu \mathrm{m}$ fluxes, so we take the median value of $\alpha$ found by the code for the $70 \mu \mathrm{m}$ subsample. We choose $\alpha$ in the interval $[1 ; 2.5]$ to cover a large domain of activity. The activity increases as $\alpha$ decreases.

\subsection{Active galactic nuclei contribution}

LIRGS have large $L_{\text {dust }}$ corresponding to an active phase of dust-enshrouded star formation and/or AGN activity. The energetic balance between dust-enshrouded stellar emission and reemission in the IR can be violated if dust emission caused by an AGN is present. In particular, highly dust-eshrouded AGNs represent a problem since they are difficult to identify in the UV and optical. Consequently, galaxies with such an AGN contribution may look like normal star-forming galaxies in the FUV-toNIR wavelength range, except for a warm/hot dust emission in the MIR.

In this paper we are interested in the $M_{\star}$ building by LIRGs. Therefore it is essential to separate the contribution of AGN and starbursts to $L_{\text {dust }}$. The AGN contribution is likely to be dominant only at very high luminosity $\left(L_{\text {dust }} \gtrsim 2 \times 10^{12} L_{\odot}\right.$, 


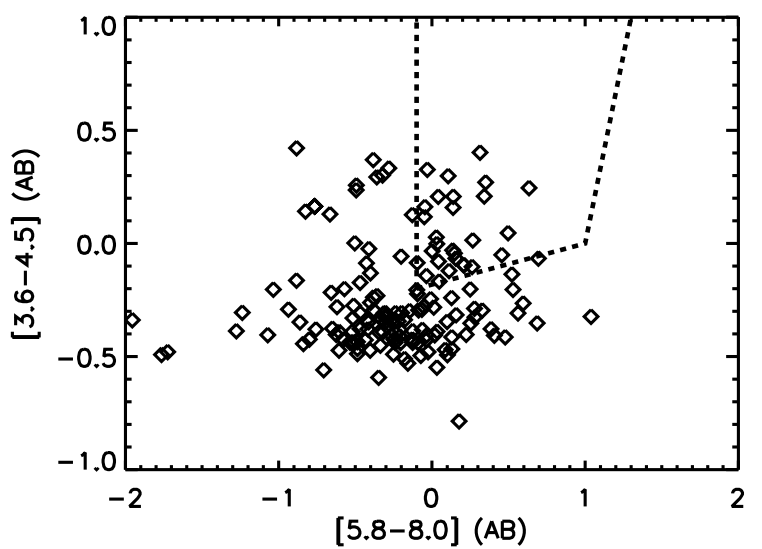

Fig. 2. Colour-colour diagram recommended by Stern et al. (2005) to identify AGN candidates. We compare the colour [ $3.6 \mu \mathrm{m}-4.5 \mu \mathrm{m}]$ versus [5.8 $\mu \mathrm{m}-8.0 \mu \mathrm{m}]$ in $\mathrm{AB}$ magnitudes for our LIRGs; the area defined by the dashed lines represents the region of the colour-colour diagram populated by AGN.

Sanders \& Mirabel 1996). Nevertheless, a small fraction of the total IR luminosity can be explained by an AGN even when dominated by star formation (e.g. Genzel et al. 1998).

To disentangle IR dust emission components caused by stellar and AGN radiation, we applied the diagnostics of Stern et al. (2005) to our observations. They combine spectroscopic observations with MIR observations of nearly 10000 sources at $0<$ $z<2$ and show that MIR photometry provides a robust technique for especially identifying broad-line AGN (90\% of broad-line AGN and $40 \%$ of narrow-line AGN are identified).

Figure 2 shows the colour-colour distribution proposed by Stern et al. (2005) in the NIR, including the boundary of the area that characterises AGN features. Fifteen percent of the objects are found within this area; however, these colour criteria may omit AGNs at $z \approx 0.8$ and 2 (Stern et al. 2005).

We also use the diagnostics of Brand et al. (2006) who examine the MIR slope $\zeta$ of the SEDs for their sample. Here, $\zeta$ corresponds to the observed flux ratio $\log _{10}\left(v f_{v}(24 \mu \mathrm{m}) / v f_{v}(8 \mu \mathrm{m})\right)$. The MIR slope is supposed to be steeper for starburst-dominated sources. Brand et al. (2006) assume that sources $(z>0.6)$ with $\zeta$ peaking at $\approx 0.0$ and $\approx 0.5$ are AGN- and starburst-dominated, respectively. Figure 3 shows the flux ratio $\zeta$ for the total sample. For the vast majority of objects, $\zeta$ is found around 0.5 ( $\sim 17 \%$ satisfies $\zeta \approx 0.0$ ), which leads us to think that starbursts dominate the MIR emission of our sample. We overplot the histogram of the objects previously identified as AGNs by Stern et al. (2005). We find that they are not necessarily identified by Brand et al. (2006).

CIGALE allows estimating the fraction of $L_{\text {dust }}$ due to an AGN. The code uses AGN templates from Siebenmorgen et al. (2004b,a) who provide almost 1500 AGN models differing in the luminosity of the nonthermal source, the outer radius of a spherical dust cloud covering the AGN, and the amount of attenuation in the visual caused by clouds. All models can be fed into CIGALE. Focusing on SEDs providing PAH-free MIR emission, the number of suitable models significantly decreases. As the reference model, we take $L=10^{12} L_{\odot}, R=125 \mathrm{pc}$, and $A_{V}=32$ mag (see Fig. 4). The parameter in our runs that probes the contribution of AGN radiation to $L_{\text {dust }}$ is named $f_{\mathrm{AGN}}$ for fraction of AGN. As input we propose values for $f_{\mathrm{AGN}}$ ranging from 0.0 to 0.999. The results are presented in Sect. 4.2.

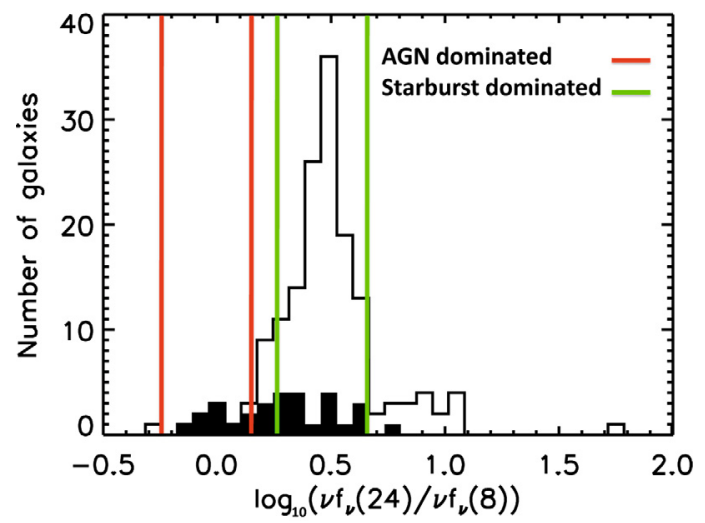

Fig.3. The empty histogram illustrates the flux ratio $\zeta=$ $\log _{10}\left(v f_{v}(24 \mu \mathrm{m}) / v f_{v}(8 \mu \mathrm{m})\right)$ for the total sample. The black histogram shows the galaxies identified as AGNs by the diagnostic of Stern et al. (2005).

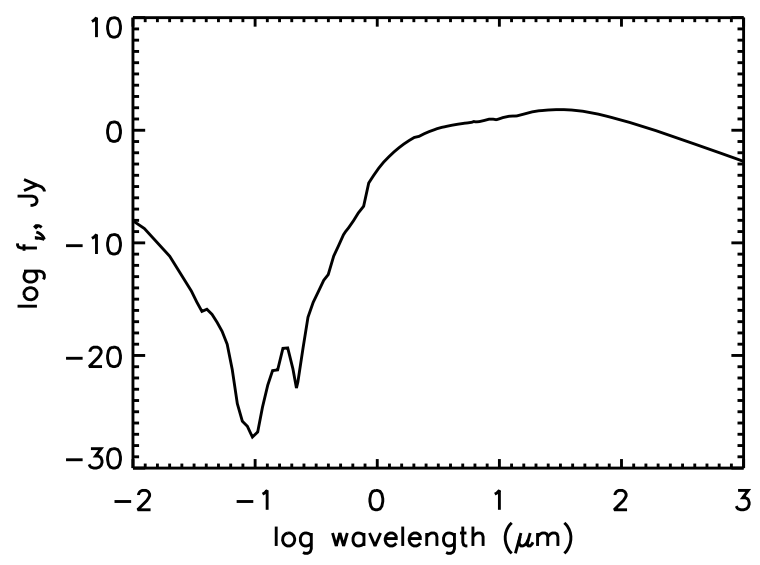

Fig. 4. SED of the adopted AGN template, with $L=10^{12} L_{\odot}, R=$ $125 \mathrm{pc}$, and $A_{V}=32 \mathrm{mag}$.

Later we will decide to flag objects identified as AGN by at least 2 diagnostics (Stern et al. 2005; Brand et al. 2006; CIGALE).

\subsection{Do we retrieve reliable parameters? Construction of a mock catalogue dedicated to the sample}

The code gives an estimate of the basic input parameters, along with several additional output parameters that depend on different basic model properties. We mostly focus on SFH parameters for the young stellar population.

We performed a Bayesian analysis to estimate the physical parameters. We used a method similar to the one of Kauffmann et al. (2003a). They derive a probability distribution function (PDF) for each parameter and estimate the most probable value. We use the mean value of this distribution. The expectation value of each parameter $\langle x\rangle$ is given as

$\langle x\rangle=\frac{\sum_{i=1}^{b} P_{i} x_{i}}{\sum_{i=1}^{b} P_{i}}$,

and the standard deviation $\sigma$ is derived by:

$\sigma_{x}=\sqrt{\frac{\sum_{i=1}^{b} P_{i}\left(x_{i}-\langle x\rangle\right)}{\sum_{i=1}^{b} P_{i}}}$. 
This method is described as the "sum" method in Noll et al. (2009b).

When we use a numerical code based on SED-fitting, we obtain results depending on a statistical analysis. The accuracy of the estimates is directly dependent on the input fluxes (number and quality). We must check which parameter the code is able to estimate correctly. Theoretically the Bayesian analysis provides an estimation of the reliability of each parameter thanks to the shape of their PDF (if the shape is not a Gaussian curve the parameter is not correctly determined; Walcher et al. 2008), and their relative error $\sigma_{x}$.

A more straightforward approach to checking the parameter estimation is to generate a mock catalogue from the real sample. Our general strategy is to build a specific mock catalogue for each set of data (in this paper the LIRGs sample). It is made of artificial galaxies for which we not only know the flux at each wavelength, but also values of attenuation parameters and parameters of the SFH.

To construct our mock catalogue we follow 3 steps. The first step is to run the code on the data to determine the best-fitting model for each object by a simple $\chi^{2}$ minimization method. Each corresponds to an SED. In the second step we add an error randomly distributed according to a Gaussian curve with $\sigma=0.1$ to each flux; it means that we add an error to the flux that is typically $10 \%$ of the flux. We have a catalogue of artificial galaxies, detected in the same filters as the observed galaxies. Then the last step is to run the code on these simulated data and to compare the exact values of the physical parameters with the values estimated by the code.

\section{Results of the SED-fitting analysis}

We performed SED-fitting on our LIRGs sample and on the related mock catalogue to know which parameter we are able to correctly estimate. In the following sections we describe the results of the SED-fitting of the mock catalogue with and without the band at $70 \mu \mathrm{m}$, and then we discuss results of the SED-fitting of the real data.

\subsection{Analysis of the mock catalogue}

We constructed two mock catalogues as described in Sect. 3.4 based on the $70 \mu \mathrm{m}$ sample (sample detected at $70 \mu \mathrm{m}$ ) only. The first catalogue is made of galaxies detected at $70 \mu \mathrm{m}(70 \mu \mathrm{m}$ mock sample), and the second one is made with the same galaxies for which we remove the flux at $70 \mu \mathrm{m}$ (mock sample) in order to know if having $70 \mu \mathrm{m}$ flux has an influence on the determination of the parameters of the SFH, or $M_{\star}, L_{\text {dust }}$ and $S F R$.

In Fig. 5 the difference is plotted in magnitudes between the flux from the best $\chi^{2}$ model estimated by CIGALE and the observed flux for each broad band filter. Mean and median values are nearly equal to zero, which means that fluxes are reproduced correctly by the code to better than 0.1 mag.

Even if the code is able to reproduce the observed data correctly, we cannot settle for this result. Indeed, it is possible that some SEDs are degenerated and correspond to different parameter values. We have to compare each input parameter with its Bayesian estimation by the code. Figures 6 and 7 show the input parameters of the mock galaxies versus the values estimated by the code. Figure 6 (Fig. 7) shows results for the $70 \mu \mathrm{m}$ mock sample (mock sample). Values of the linear Pearson correlation coefficient $r$ are gathered in Table 3 .

For the $70 \mu \mathrm{m}$ mock sample, we find very good correlations for $M_{\mathrm{star}}, S F R, L_{\mathrm{dust}}, f_{\mathrm{AGN}}$, and $A_{V}$ with $r>0.9$. For $t_{2}$,

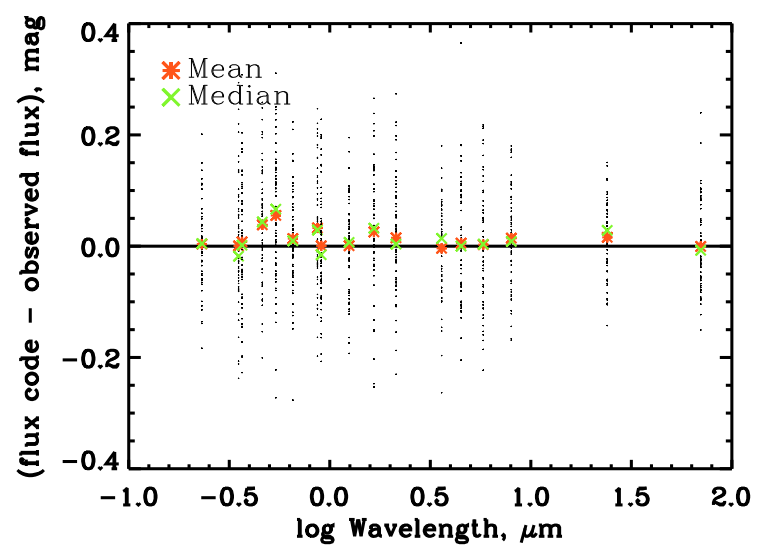

Fig. 5. Difference in magnitudes between the flux of the mock galaxies and the flux of the best $\chi^{2}$ model for these objects in each band. Red crosses and green crosses represent the mean and the median value for each wavelength.

Table 3. Estimation of the linear correlation coefficient of Pearson between the exact value and the value estimated by CIGALE for each parameter of the mock catalogue.

\begin{tabular}{lll}
\hline \hline Parameters & $\mathbf{7 0} \mu$ m mock sample & mock sample \\
\hline $\log _{10} M_{\text {star }}$ & 0.93 & 0.92 \\
$\log _{10} L_{\text {dust }}$ & 0.93 & 0.95 \\
$\log _{10} S F R$ & 0.93 & 0.92 \\
$t_{2}$ & 0.34 & 0.20 \\
$f_{\text {ySP }}$ & 0.52 & 0.45 \\
$A_{V}$ & 0.92 & 0.80 \\
$f_{V}$ & 0.64 & 0.64 \\
$\alpha$ & 0.80 & 0.04 \\
$f_{\text {AGN }}$ & 0.92 & 0.76 \\
\hline
\end{tabular}

Notes. We estimate this coefficient for the $70 \mu \mathrm{m}$ mock sample and the mock sample.

$f_{\mathrm{ySP}}$, and $f_{V}$, the correlation is less satisfying, because $r$ is equal to $0.34,0.52$, and 0.64 for the $70 \mu \mathrm{m}$ mock sample and 0.20 , 0.45 , and 0.64 for the mock sample. The correlation for the parameter $\alpha$ is good with $r=0.80$ for $70 \mu \mathrm{m}$ mock sample but very bad for the mock sample. In the following we use the mean value of $\alpha$ from the $70 \mu \mathrm{m}$ sample for the objects without detection at $70 \mu \mathrm{m}$. For $f_{\mathrm{AGN}}, r>0.9$ for $70 \mu \mathrm{m}$ mock sample and 0.76 for the mock sample, with relatively small errors. Thus this parameter is correctly estimated by the code.

According to the analysis of the coefficient $r$ and the Bayesian method's error, the reliable parameters are $M_{\text {star }}, L_{\text {dust }}$, $S F R, f_{\mathrm{ySP}}, A_{V}, \alpha$ (if $70 \mu \mathrm{m}$ is available) and $f_{\mathrm{AGN}}$. Consequently in the next section we only study these parameters.

\subsection{Analysis of the LIRGs sample}

We now present the results for the LIRGs sample itself. Figure 8 shows distributions for the parameters of interest, calculated with the Bayesian analysis described previously for the subsample detected at $70 \mu \mathrm{m}$ and for the whole sample. The masses found for the $70 \mu \mathrm{m}$ sample are shifted towards higher masses then in the total sample. We observe a similiar situation for $L_{\text {dust }}$ and the SFR; the values are in the range $\left[10^{11} ; 10^{12}\right] L_{\odot}$ and [10; 92] $M_{\odot} \mathrm{yr}^{-1}$, respectively, with mean values higher than ones found for the total sample. This shift is expected because of the $70 \mu \mathrm{m}$ detection limit $(2.7 \mathrm{mJy}$ at $5 \sigma)$ : at this wavelength, we only detect luminous and massive galaxies. The distribution 
E. Giovannoli et al.: LIRGs at intermediate redshift
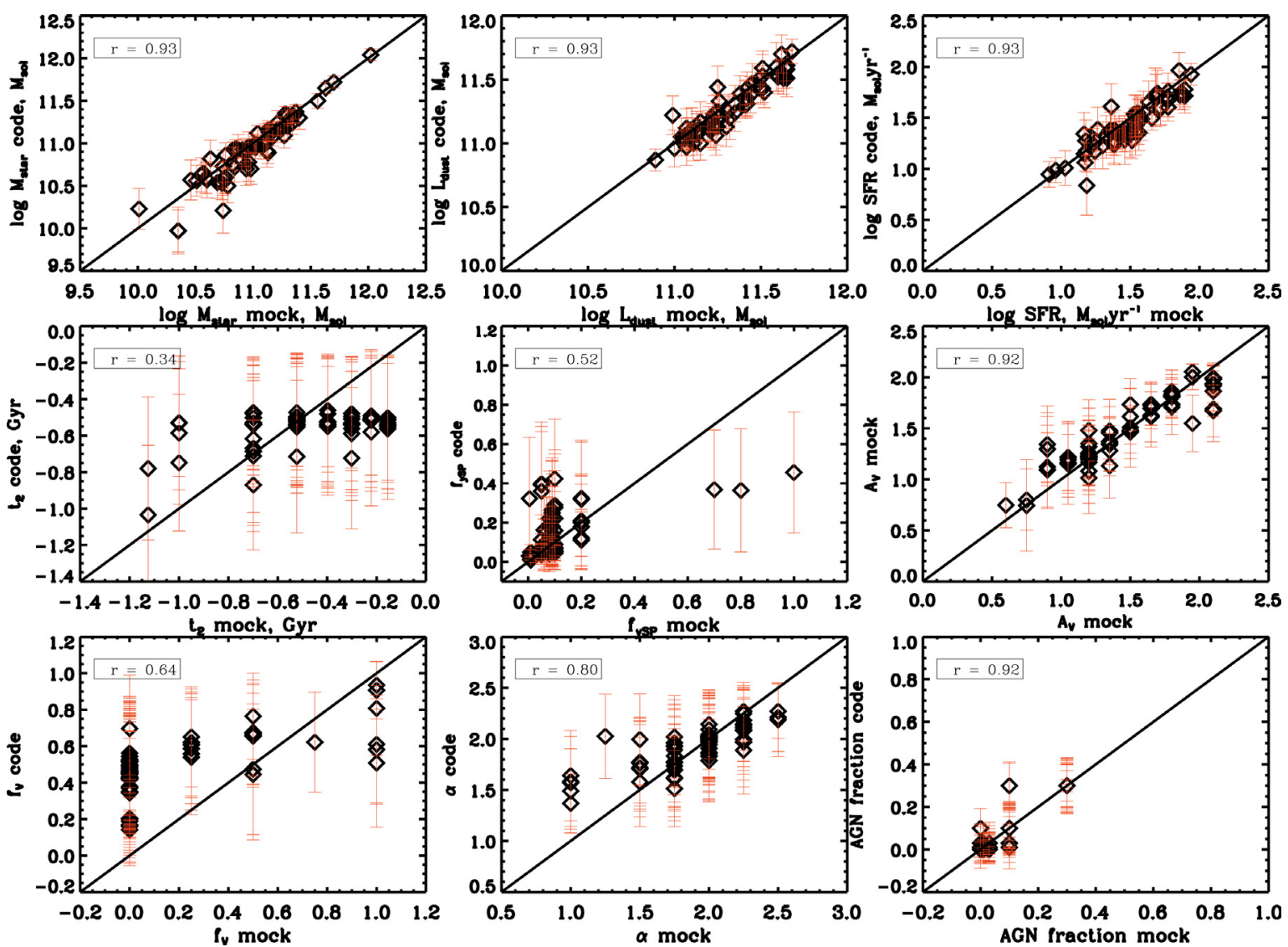

Fig. 6. On the $x$ axis, the value for 8 parameters of the mock galaxies in the case of a detection at $70 \mu \mathrm{m}$, and on the y axis their values estimated by CIGALE. The black line corresponds to the ratio 1:1.
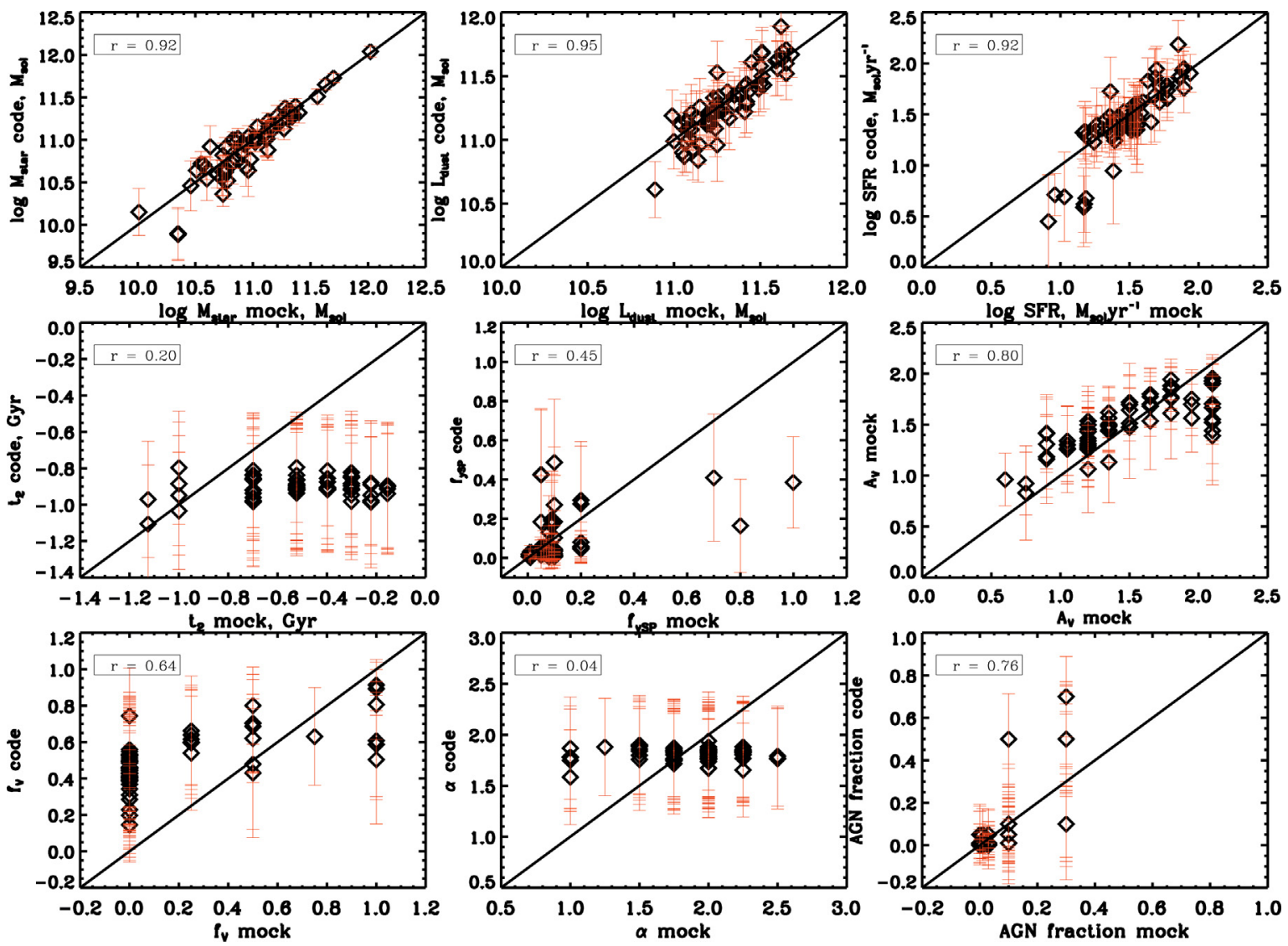

Fig. 7. Same plot as Fig. 6 in the case of non-detection at $70 \mu \mathrm{m}$. 

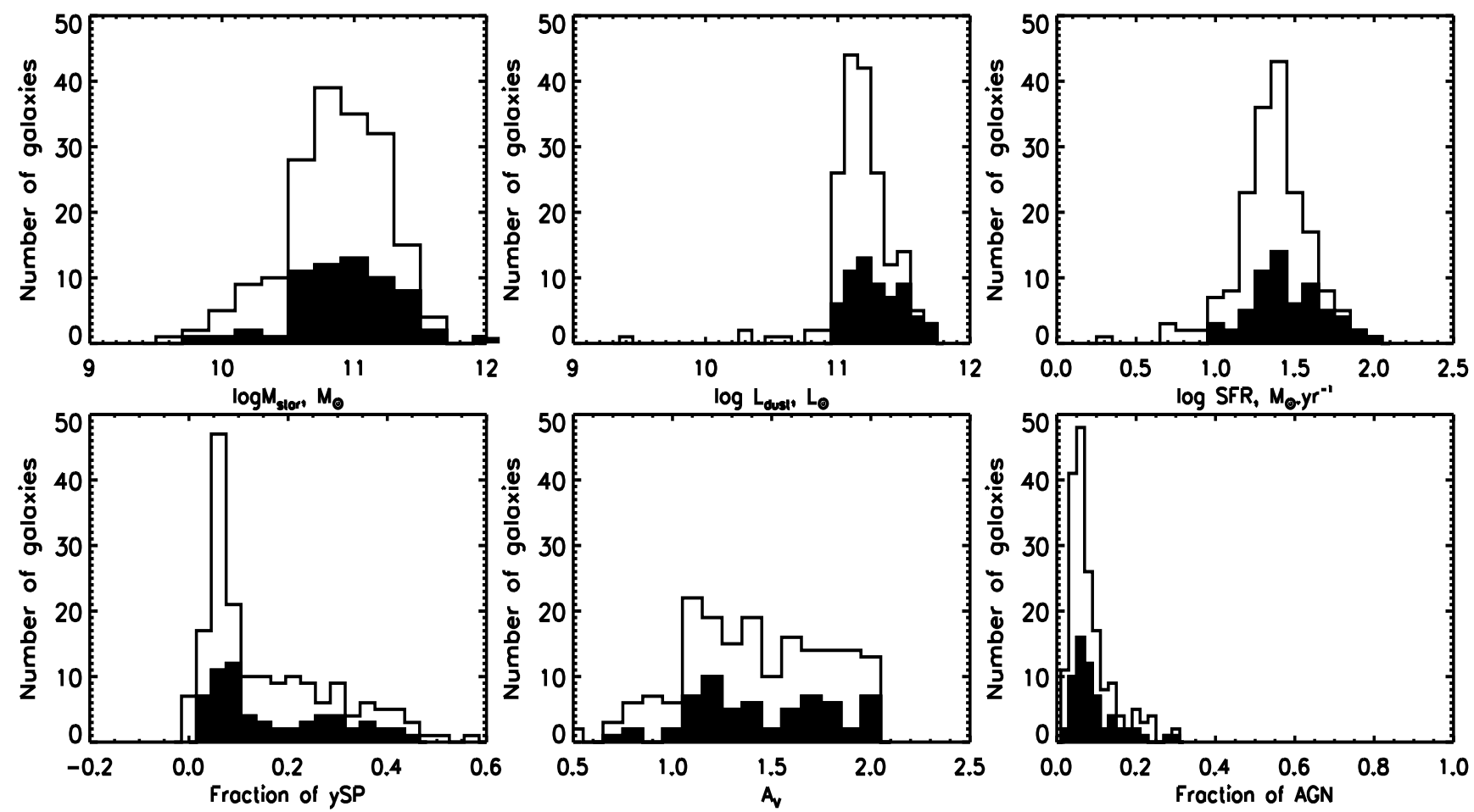

Fig. 8. Bayesian results of the code for the following parameters: $M_{\mathrm{star}}, L_{\mathrm{dust}}, S F R, A_{V}, f_{\mathrm{ySP}}$, and $f_{\mathrm{AGN}}$. The empty histogram represents the total sample and the full one represents the $70 \mu \mathrm{m}$ sample.

of $f_{\mathrm{ySP}}$ is broad with a long tail towards high values, and $A_{V}$ lies between 0.5 and $2.1 \mathrm{mag}$ with very few objects under $1.0 \mathrm{mag}$ and quite a homogeneous distribution between 1.0 and 2.0 .

Galaxies in the total sample have $M_{\star}$ between $10^{10}$ and $10^{12} M_{\odot}$ with a peak at $10^{10.8} M_{\odot}$. We find the SFR between 3 and $92 M_{\odot} \mathrm{yr}^{-1}$ with a peak at $23 M_{\odot} \mathrm{yr}^{-1}$. For $f_{\mathrm{ySP}}$ and $A_{V}$, we observe the same distribution as for the $70 \mu \mathrm{m}$ sample. For both samples, $f_{\mathrm{AGN}}$ is relatively low, between 0.0 and 0.3 with the majority of the objects in the interval $[0 ; 0.1]$. We consider that there is a definite contamination of $L_{\text {dust }}$ by an AGN when $f_{\mathrm{AGN}}>15 \%$, because a contamination lower than $15 \%$ does not significantly modify the total IR emission. We must also account for the uncertainty of the $f_{\mathrm{AGN}}$ determination: we only consider as AGN contaminated objects with $f_{\mathrm{AGN}}>15 \%$ and $f_{\mathrm{AGN}}-\sigma_{\mathrm{AGN}}>0$ where $\sigma_{\mathrm{AGN}}$ is the standard deviation (see Eq. (5)).

We identify 21 objects in the total sample. The diagnostics of Stern et al. (2005) identify 31 objects and Brand et al. (2006) 9 objects. However, all the objects considered as AGN by the code are not systematically identified as AGN in the diagnostics of Brand et al. (2006) and Stern et al. (2005). We consider objects as contaminated by AGN when they are flagged by two of the three diagnostics. We identify 15 objects in the total sample of which 6 are in the $70 \mu \mathrm{m}$ sample. These objects will be flagged in the following plots.

We have also investigated the small subsample of galaxies not detected in NUV (31 over 181 galaxies) and did not find any statistically significant difference in their main characteristics. The largest effect is found for $A_{V}: A_{V}=1.75+/-0.25$ mag for the non detections in NUV and $A_{V}=1.33+/-0.34$ mag for the detections in NUV. A higher dust attenuation for galaxies not detected in NUV is indeed expected.

\section{Discussion}

\subsection{Luminosity-colour distribution and the implication for the dust temperature}

SED shape of an LIRG in the $8-1000 \mu \mathrm{m}$ range is due to emission from dust. The SED rest-frame peak defines the wavelength of maximum energetic output (in $v f_{v}$ ). It is usually in the $40-140 \mu \mathrm{m}$ range, and the SED is often approximated by a modified black-body $\left(B_{v} \nu^{\beta}\right)$ of $T \sim 20-60 \mathrm{~K}$ and emissivity $\beta \sim 1-2$.

Local IRAS (Infrared Astronomical Satellite) galaxies exhibit correlations between the colour $f_{60 \mu \mathrm{m}} / f_{100 \mu \mathrm{m}}$ and $L_{\text {dust }}$ (e.g. Dale et al. 2001). The $f_{60 \mu \mathrm{m}} / f_{100 \mu \mathrm{m}}$ ratio is often taken as an indicator of the typical heating conditions in the interstellar medium and is also indicative of a characteristic dust temperature (hereafter $T_{\text {dust }}$ ), $f_{60 \mu \mathrm{m}} / f_{100 \mu \mathrm{m}}$ increasing with $T_{\text {dust }}$.

In the nearby universe, LIRGs are characterised by emission from dust at high temperatures (Sanders \& Mirabel 1996). Fitting single $T_{\text {dust }}$ to $f_{60} \mu \mathrm{m} / f_{100 \mu \mathrm{m}}$ ratio leads to $T_{\text {dust }} \sim 20 \mathrm{~K}$ for low-redshift spirals (Reach et al. 1995; Dunne \& Eales 2001) and 30-60 K for the high-luminosity objects typically detected by IRAS (Soifer \& Neugebauer 1991; Stanford et al. 2000). Highredshift, hyperluminous galaxies can show $T_{\text {dust }}$ of up to $110 \mathrm{~K}$ (Lewis et al. 1998).

To reproduce number counts at FIR wavelengths, Lagache et al. (2003), Chapin et al. (2009a), and Valiante et al. (2009) find that it is necessary to take cold luminous galaxies into account in the nearby universe. Cold galaxies are found to dominate the $z=0$ luminosity function at low luminosities and become less important at higher redshifts, because of their passive evolution. The fraction of cold galaxies can contribute to the number counts up to $50 \%$ at $170 \mu \mathrm{m}$. At moderate and high redshift, the possible importance of cold, luminous galaxies has also been pointed out by Eales et al. (1999) and Chapman et al. (2002). 




Fig. 9. Variations of $f_{14 \mu \mathrm{m}} / f_{41 \mu \mathrm{m}}$ versus $f_{60 \mu \mathrm{m}} / f_{100 \mu \mathrm{m}}$ (expressed in Jansky) for the DH02 templates used in the paper, corresponding to the $\alpha$ range [1.0-2.5].

We use the fluxes ratio $f_{24} \mu \mathrm{m} / f_{70 \mu \mathrm{m}}$ (corresponding to the highest wavelengths in our sample) to estimate the dust temperature. The observed $f_{24} \mu \mathrm{m} / f_{70 \mu \mathrm{m}}$ corresponds to $f_{14 \mu \mathrm{m}} / f_{41 \mu \mathrm{m}}$ restframe. All our discussions are made in the framework of the DH02 models in the [1.0-2.5] $\alpha$ regime (see Table 2), and $f_{14 \mu \mathrm{m}} / f_{41 \mu \mathrm{m}}$ evolves in the opposite way of $f_{60 \mu \mathrm{m}} / f_{100 \mu \mathrm{m}}$ (i.e. $T_{\text {dust }}$, see Fig. 9).

Figure 10 exhibits the observed $f_{24} \mu \mathrm{m} / f_{70 \mu \mathrm{m}}$ ratio as a function of $L_{\text {dust }}$ obtained for the LIRGs in the $70 \mu \mathrm{m}$ sample. Predictions of DH02 models are overplotted. They are calibrated with the Marcillac et al. (2006) local relation between $f_{60 \mu \mathrm{m}} / f_{100 \mu \mathrm{m}}$ (i.e. $\alpha$ ) and $L_{\text {dust }}$. For a given $L_{\text {dust }}$ we find that our galaxies have a $f_{14 \mu \mathrm{m}} / f_{41} \mu \mathrm{m}$ ratio higher than predicted by the models. This implies that they have a lower $f_{60 \mu \mathrm{m}} / f_{100 \mu \mathrm{m}}$ ratio and are thus colder than expected. We cannot attribute this result to the presence of AGN contaminated objects regarding the small fraction of flagged sources.

We can also directly use the $f_{60} \mu \mathrm{m} / f_{100 \mu \mathrm{m}}$ ratio deduced from the $\alpha$ parameter estimated by CIGALE. In Fig. 11 we compare $L_{\text {dust }}$ and the derived $f_{60} \mu \mathrm{m} / f_{100 \mu}$ m values of our $70 \mu \mathrm{m}$ sample. It will make no sense to compare the rest of the sample for which we estimated $\alpha$ with the median value of $\alpha$ found for the subsample. We add to this plot the local $L_{\text {dust }}-f_{60} \mu \mathrm{m} / f_{100 \mu \mathrm{m}} \mathrm{re}-$ lations of Chapman et al. (2003) and Marcillac et al. (2006), and the luminosity-temperature models proposed by CE01, Chapin et al. (2009a), and Valiante et al. (2009) in the luminosity range $\left[10^{11} ; 10^{12}\right] L_{\odot}$. Figure 11 shows that the code gives a different trend for the relation $f_{60 \mu} / f_{100 \mu \mathrm{m}}$ versus $L_{\text {dust }}$ than both local relations and models. Galaxies having the lowest IR luminosity agree with them, but more luminous objects are found to be colder than predicted, and globally they evolve in the opposite way to local relations and models. Chanial et al. (2007) make a selection at 60 and $170 \mu \mathrm{m}$ respectively using IRAS and ISO observations at $z=0$ and find that galaxies selected at $170 \mu \mathrm{m}$ are colder than ones detected at shorter wavelengths. They conclude that selecting galaxies at long wavelengths introduce a bias towards cold galaxies. Selecting the sample at a wavelength longwards of the peak, by definition, does not lead to warm sources (Sanders \& Mirabel 1996). Our sample is selected by restframe $\sim 41 \mu \mathrm{m}$ emission, i.e., on warm dust; yet, we mostly find colder $T_{\text {dust }}$ at a given luminosity than predicted by models of DH02 and CE01, calibrated in the nearby universe. This tends to support the interpretation that the offset found towards colder temperatures at a given luminosity is at least in part a real difference. Zheng et al. (2007) suggest that this tendency towards colder $T_{\text {dust }}$ reflects a difference in dust and star formation geometry. Although local LIRGs tend to be interacting systems with relatively compact very intense star formation and of comparable masses (e.g., Wang et al. 2006), $0.5<z<1$ LIRGs tend to be disk-dominated, relatively undisturbed galaxies (Zheng et al. 2004; Bell et al. 2005; Melbourne et al. 2005). They suggest that these diskgalaxies host widespread, intense star formation, much like star formation in local spirals but scaled up, leading to relatively cold $T_{\text {dust }}$.

Our results agree with Symeonidis et al. (2009). They compare FIR properties of LIRGs and Ultra Luminous InfraRed Galaxies (ULIRGs, $L_{\text {dust }}>10^{12} L_{\odot}$ ) in the $0.1<z<2$ redshift range, based on MIPS and IRAC data with $L_{\text {dust }}>10^{10} L_{\odot}$, $70 \mu \mathrm{m}$ selected objects, with those of local sources of equivalent luminosity from the local IRAS Bright Galaxy Sample. They fit their data with the Siebenmorgen \& Krügel (2007) theoretical SED templates and find that their distant LIRGs and ULIRGs are colder than their local equivalents. They find a significant cold-dust associated FIR excess not detected in local sources of comparable luminosity directly linked to the evolution in dust and star formation properties from the local to the high-redshift Universe.

Herschel is now providing longward $70 \mu \mathrm{m}$ data allowing a reliable estimation of $T_{\text {dust }}$.

\subsection{Star formation rate and stellar mass}

How and when galaxies build up their $M_{\star}$ is still a major question in observational cosmology. While a general consensus has been reached in recent years on the evolution of the galaxy stellar mass function (e.g., Dickinson et al. 2003; Drory et al. 2004; Bundy et al. 2005; Pannella 2006; Marchesini et al. 2009), the redshift evolution of the SFR as a function of $M_{\star}$ still remains unclear. The evolution of the galaxy $S F R-M_{\star}$ relationship provides key constraints on $M_{\star}$ assembly histories of galaxies. For star-forming galaxies a correlation is observed between SFR and $M_{\star}$ with a slope near to unity from $z \sim 0$ to 2 and a scatter of 0.3 dex (Daddi et al. 2007; Elbaz et al. 2007; Noeske et al. 2007a). The slope and scatter are expected to be a direct reflection of the similarity in the shape of galaxy SFHs among various models and at various masses (Davé 2008; Noeske et al. 2007b). It is worth noting that other $z=2$ observations suggest a somewhat large scatter (Shapley et al. 2005; Papovich et al. 2006), so the observational picture is not entirely settled.

Figure 12 shows the SFR of each galaxy of our sample as a function of the corresponding $M_{\star}$ and the results of various models and observations: analytical models of Buat et al. (2008) and Noeske et al. (2007a), the semi-analytical Millennium simulations of Kitzbichler \& White (2007) used in Buat et al. (2008) and observations of Elbaz et al. (2007), Daddi et al. (2007), and Santini et al. (2009). Our sample of LIRGs has an average redshift of $\sim 0.7$. We find the vast majority of the sample between the correlations at $z=0$ and $z=1$ given by Elbaz et al. (2007). Our data cover the extreme part of the Millennium simulations where we can find galaxies with the largest SFRs between $\sim 10-100 M_{\odot} \mathrm{yr}^{-1}$. We do not observe a correlation between $S F R$ and $M_{\star}$, but instead a flat distribution of SFR. We consider a tight range of SFR since we select objects with $L_{\text {dust }} \geqslant 10^{11} L_{\odot}$ (see below). This range is not sufficient to search for a general correlation between $M_{\star}$ and the SFR; however, we observe a large scatter in $M_{\star}$ in the considered SFR range. Indeed galaxies whose young stellar population is younger than $300 \mathrm{Myr}$ exhibit a larger dispersion in $M_{\star}$ than galaxies whose SFR is constant 


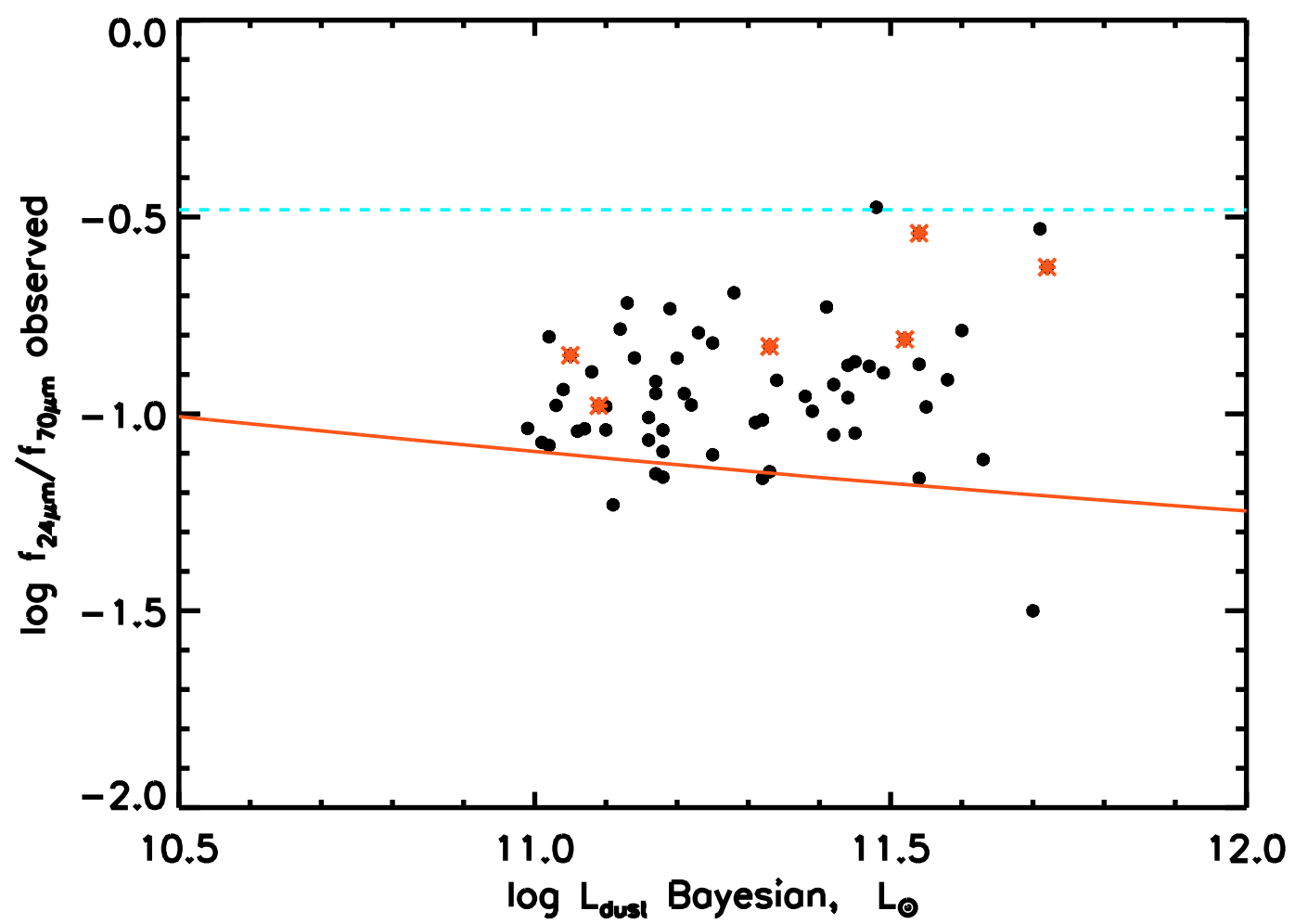

Fig. 10. This figure illustrates the ratio between the observed fluxes at $24 \mu \mathrm{m}$ and $70 \mu \mathrm{m}$ as a function of the $L_{\text {dust }}$ estimated by the code. The solid red line corresponds to the results given by the DH02 templates at $z=0.7$. The dashed cyan line is given by Siebenmorgen et al. (2004b) for typical AGN contaminated object. AGNs described in Sect. 4.2 are shown as red stars.

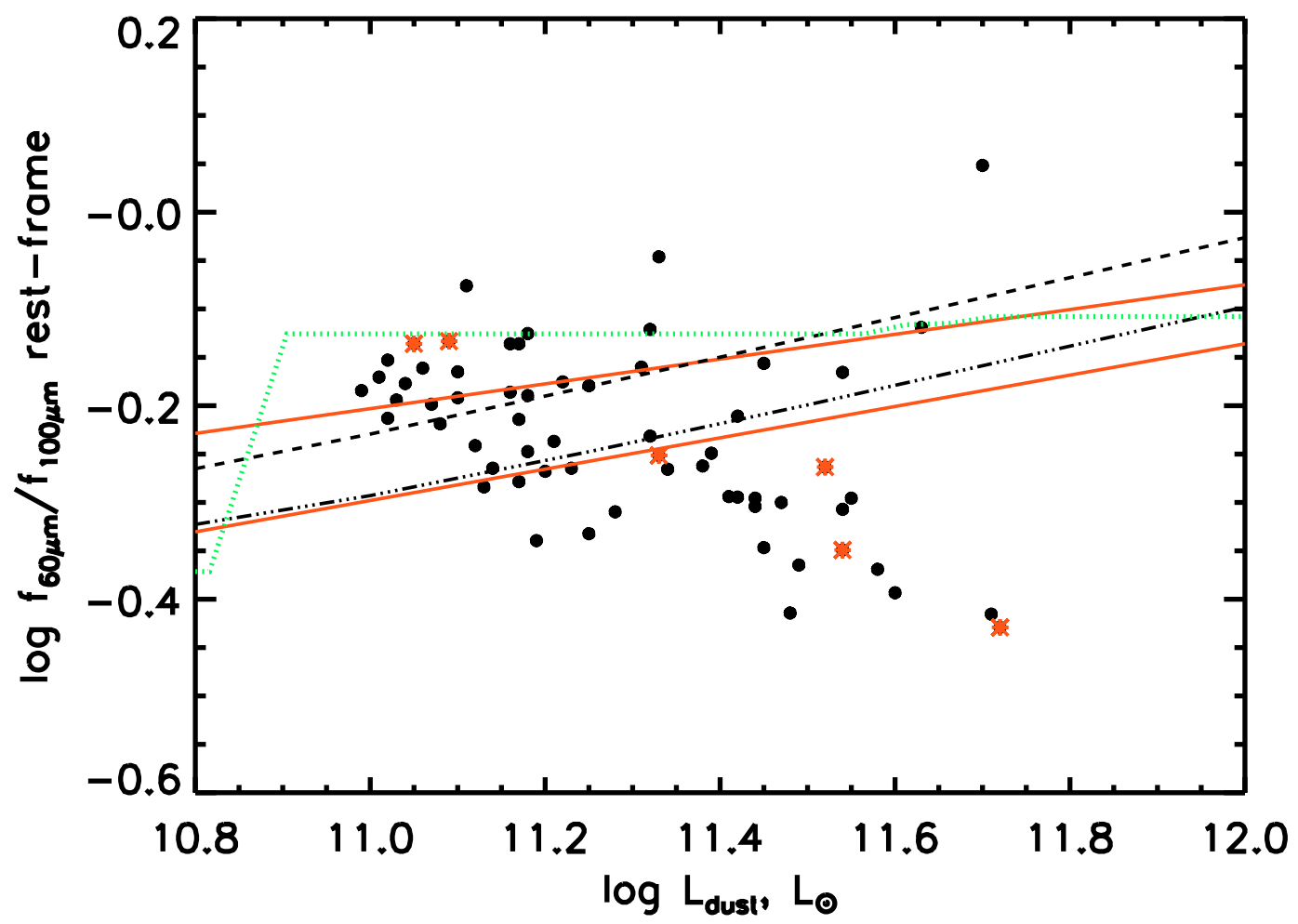

Fig. 11. Comparaison of the relation $\log _{10}(\mathrm{f} 60 \mu \mathrm{m} / \mathrm{f} 100 \mu \mathrm{m})-\log _{10} L_{\text {dust }}$ given by IR SEDs of DH02 after the fit to the data, with the values given by local relations and models. Upper and lower solid red lines represent local relations of Chapman et al. (2003) and Marcillac et al. (2006), respectively. We overplot models of CE01 (green dotted line), Chapin et al. (2009a) (dashed black line), and Valiante et al. (2009) (dash-dotted black line). AGN detections are shown as red stars.

over a longer period (Fig. 12), therefore the SFH of our objects has an influence on the dispersion of the $S F R-M_{\star}$ plot. This agrees with the conclusions of Davé (2008) and Noeske et al. (2007b). 
E. Giovannoli et al.: LIRGs at intermediate redshift

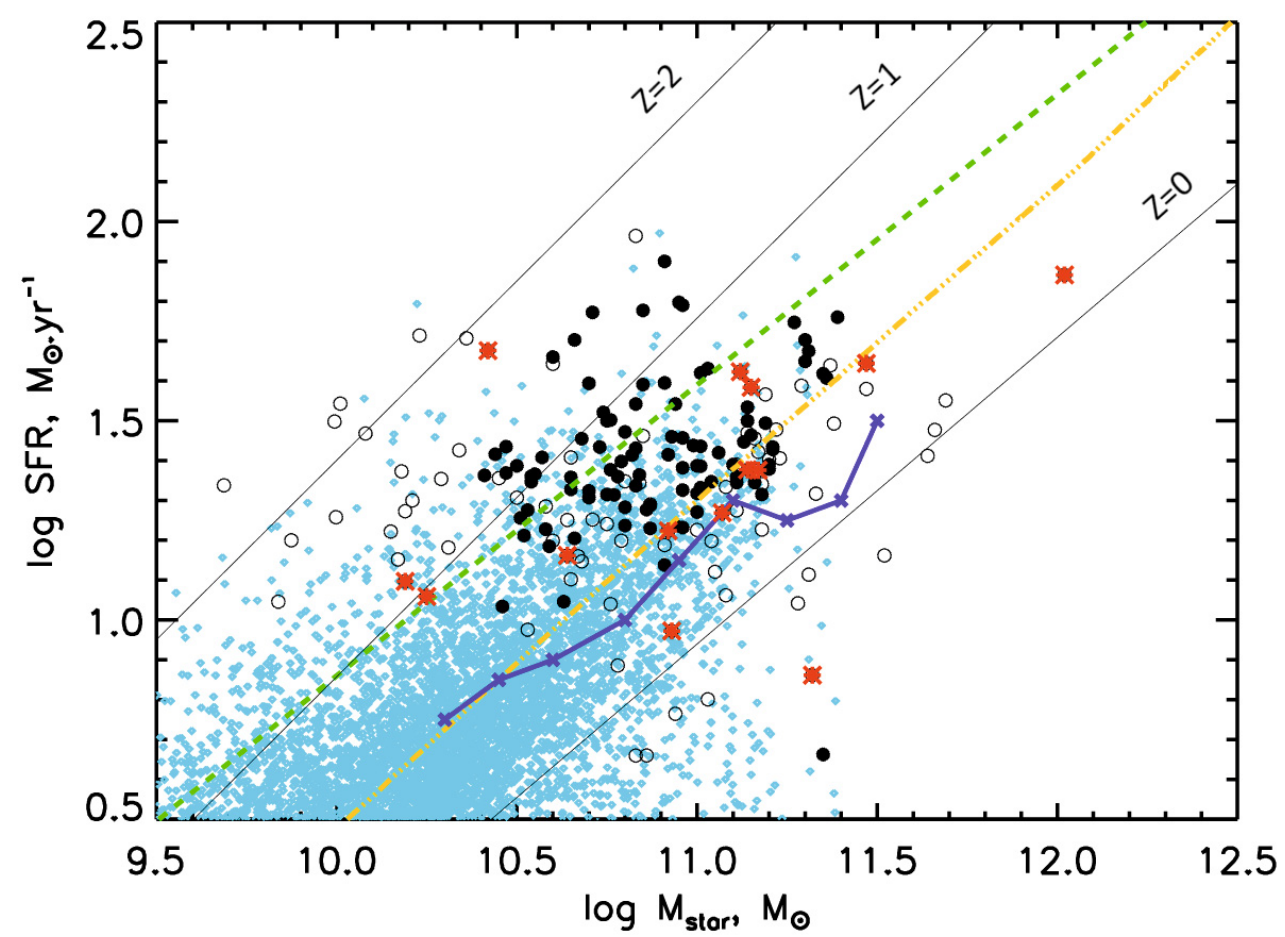

Fig. 12. The relation $S F R-M_{\star}$ for the total sample. Filled circles (empty circles) illustrate galaxies for which the age of the young stellar population is found to be greater (lower than or equal to) than 0.3 Gyr by CIGALE. AGNs are represented by red stars. We overplot the semianalytical Millennium simulations (cyan diamonds), analytical models of Buat et al. (2008) (dash-dotted yellow line) at $z=0.7$ and Noeske et al. (2007a) (connected blue crosses) at $z \sim 0.7$ with observations of Elbaz et al. (2007) (solid black line, $z=0$ and 1), Daddi et al. (2007) (solid black line $z=2$ ), and Santini et al. (2009) at $z \sim 0.7$ (dashed green line).

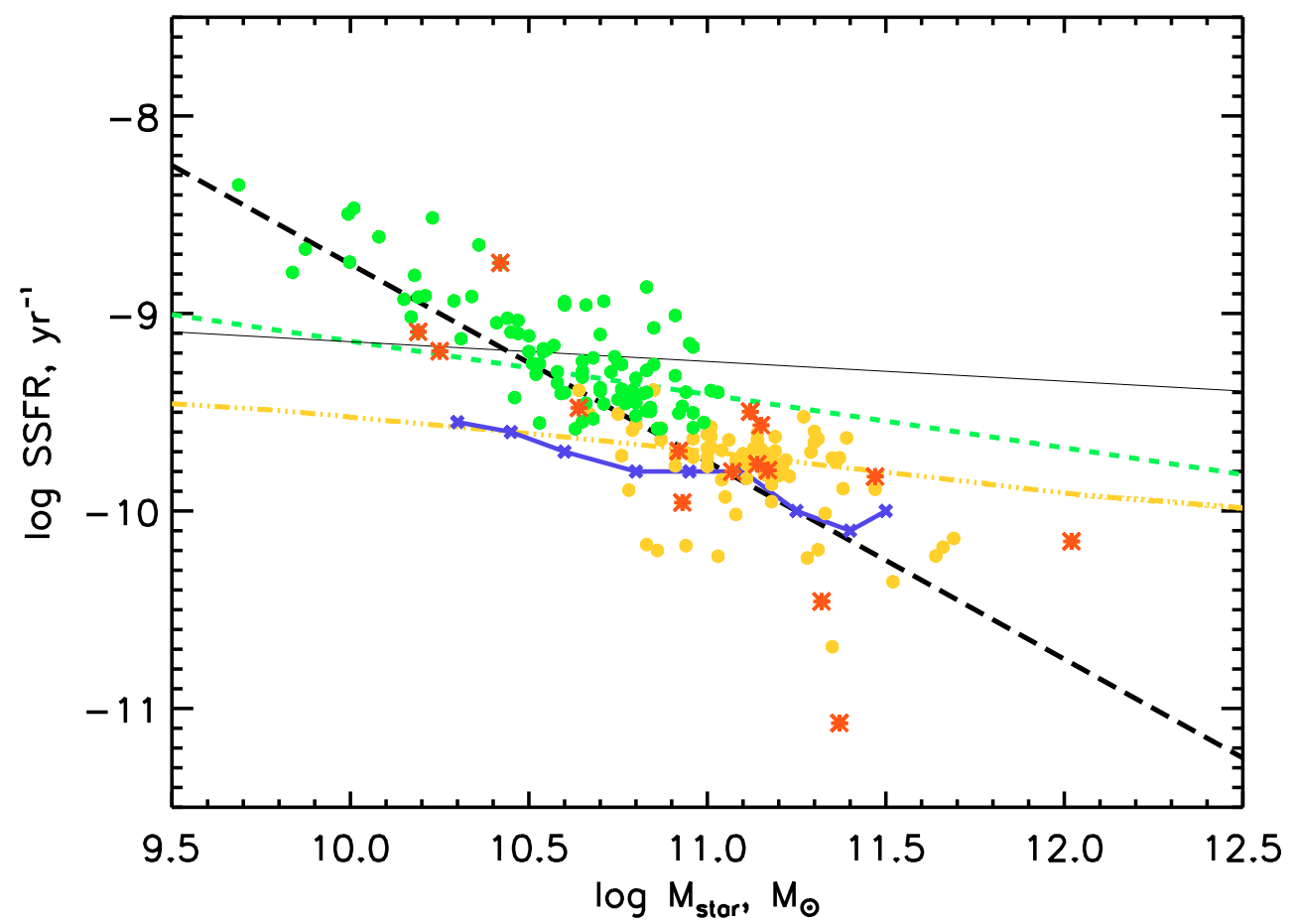

Fig. 13. The $S S F R-M_{\star}$ relation for the total sample. Green dots and yellow dots represent objects for which $f_{\mathrm{ySP}}$ are larger or lower than $10 \%$, respectively. AGN contaminated objects are represented by red stars. The black dashed line shows the detection limit. We overplot analytical models of Buat et al. (2008) (dash-dotted yellow line) at $z=0.7$ and Noeske et al. (2007a) (connected blue crosses) at $z \sim 0.7$, and observations of Elbaz et al. (2007) (solid black line, $z=1$ ) and Santini et al. (2009) at $z \sim 0.7$ (dashed green line).

The specific star formation rate (SSFR) defined as the ratio between $S F R$ and $M_{\star}$ (e.g., Kennicutt et al. 2005) is also commonly used to trace the SFH in galaxies. The SSFR is plotted in Fig. 13 as a function of the corresponding $M_{\star}$, and we can see the increase in the SSFRs towards lower $M_{\star}$. Before any interpretation we can estimate a detection limit in 
SSFR: $L_{\text {dust }}=10^{11} L_{\odot}$ translates into $S F R_{\text {lim }}=17.78 M_{\odot} \mathrm{yr}^{-1}$ at $z=0.7$ (Eq. (1) of Buat et al. 2008). We overlay this detection limit and see that the trend of SSFR with $M_{\star}$ is largely constrained by this limit. We compare our results with the same models and observations as illustrated in Fig. 12. SSFRs of our intermediate-mass galaxies $\left(M_{\star} \leq 10^{11} M_{\odot}\right)$ agree with those observed by Santini et al. (2009), but for these values of $M_{\star}$ we cannot detect more quiescent galaxies because of the detection limit. SSFRs of high-mass galaxies $\left(M_{\star}>10^{11} M_{\odot}\right)$ are better described by the models of Buat et al. (2008). These massive galaxies have SSFRs which do not exceed $2.5 \times 10^{-10} \mathrm{yr}^{-1}$ (corresponding to a $S F R \sim 25 M_{\odot} \mathrm{yr}^{-1}$ ) and never reach the SSFRs of intermediate-mass galaxies observed in this study. To distinguish galaxies for which the young stellar population significantly contributes to $M_{\star}$, we identify objects for which $f_{\mathrm{ySP}}$ estimated with CIGALE is larger or lower than $10 \%$ and see that the 2 groups are separated well according to their stellar mass. Galaxies with $M_{\star} \leq 10^{11} M_{\odot}$ have more than $10 \%$ of their mass due to the young stellar population, whereas in galaxies with $M_{\star}>10^{11} M_{\odot}$ this mass represents less than $10 \%$. This result agrees with the common picture that massive galaxies formed the bulk of their stars early and on shorter time scales, while numerous less massive galaxies evolve on longer time scales, a phenomenon generally linked to the downsizing scenario (e.g. Cowie et al. 1996; Brinchmann \& Ellis 2000; Fontana et al. 2003; Feulner et al. 2005; Pérez-González et al. 2005; Papovich et al. 2006; Damen et al. 2009).

\section{Conclusions}

We performed an SED-fitting analysis of a sample of LIRGs at $z=0.7$ detected at $24 \mu \mathrm{m}$ by Spitzer/MIPS, for which $80 \%$ of the objects have a detection at $231 \mathrm{~nm}$ by GALEX. This sample is observed over a wide range of wavelengths from NUV to FIR $(70 \mu \mathrm{m})$ and is made of 181 galaxies with 62 detected at $70 \mu \mathrm{m}$. We fit the SEDs of our galaxies with the CIGALE code (Burgarella et al. 2005; Noll et al. 2009b), which combines stellar and dust emissions in a physical way. This study is the first use of CIGALE at a redshift higher than 0. The stellar populations synthesis code of Maraston et al. (2005) is adopted to model the stellar emission (UV, optical, and NIR wavelengths). Then the code uses semi-empirical one-parameter models of Dale \& Helou (2002) to reproduce the dust emission in MIRto-FIR wavelengths.

CIGALE allows estimation of several physical parameters based on a Bayesian-like analysis. We show that we are able to estimate reliable stellar masses, SFRs, fractions of young stellar population, Dale \& Helou (2002) IR-templates parameter $\alpha$ (if $70 \mu \mathrm{m}$ is available), dust attenuation, dust luminosities, as well as the fraction of dust emission from an AGN. The investigation is performed by building mock catalogues based on the real samples.

The $70 \mu \mathrm{m}$ sample exhibits colder dust temperatures (as traced by the ratio $L_{24} \mu \mathrm{m} / L_{70} \mu \mathrm{m}$ and the $\alpha$ parameter of the Dale \& Helou 2002 templates) than expected from local relations between dust luminosity and temperature, confirming other recent results for similar samples. Our LIRGs appear to form stars actively. They exhibit a flat distribution with a large scatter in the SFR - stellar mass plot. The amplitude of the dispersion is related to the age of the young stellar population, with a tighter distribution found for the oldest ages. We find that our galaxies with a stellar mass $>10^{11} M_{\odot}$ have less than $10 \%$ of their mass coming from the young stellar population. The SSFR for these massive galaxies never reaches the one found for intermediate-mass galaxies of our sample, confirming the downsizing scenario.

The multiwavelength data analysis performed in this study provides reliable estimates of several physical parameters, but may turn out insufficient for determining accurate dust temperatures. Forthcoming data from Herschel will help us to better constrain galaxies SEDs and thus to derive more reliable parameters.

\section{References}

Arnouts, S., Vandame, B., Benoist, C., et al. 2001, A\&A, 379, 740 Arnouts, S., Schiminovich, D., Ilbert, O., et al. 2005, ApJ, 619, L43 Arnouts, S., Walcher, C. J., Le Fèvre, O., et al. 2007, A\&A, 476, 137 Basu-Zych, A. R., Schiminovich, D., Johnson, B., et al. 2007, ApJS, 173, 457 Bauer, F. E., Alexandern, D. M., Brandt, W. N., et al. 2004, AJ, 128, 2048 Bell, E. F., MacIntosh, D. H., Katz, N., \& Weinberg, N. D. 2003, ApJS, 149, 289 Bell, E. F., Papovich, C., Wolf, C., et al. 2005, ApJ, 625, 36 Bell, E. F., Zheng, X. Z., Papovich, C., et al. 2007, ApJ, 663, 834 Brand, K., Weedman, D., Desai, V., et al. 2006, ApJ, 644, 147 Brinchmann, J., \& Ellis, R. S. 2000, ApJ, 536, 77

Brinchmann, J., Charlot, S., White, S. D. M., et al. 2004, MNRAS, 351, 1151 Bruzual, G., \& Charlot, S. 2003, MNRAS, 344, 1000

Buat, V., Deharveng, J. M., Burgarella, D., \& Kunth, D. 2002, A\&A, 393, 33

Buat, V., Iglesias-Páramo, J., Seibert, M., et al. 2005, ApJ, 619, L51

Buat, V., Takeuchi, T. T., Iglesias-Páramo, J., et al. 2007a, ApJS, 173, 404

Buat, V., Marcillac, D., Burgarella, D., et al. 2007b, A\&A, 469, 19

Buat, V., Boissier, S., Burgarella, D., et al. 2008, A\&A, 483, 107

Bundy, K., Ellis, R. S., \& Conselice, C. J. 2005, ApJ, 625, 621

Burgarella, D., Buat, V., \& Iglesias-Páramo, J. 2005, MNRAS, 1413, 1425

Burgarella, D., Pérez-González, P., Tyler, K. D., et al. 2006, A\&A, 450, 69

Burgarella, D., Le Floc'h, E., Takeuchi, T. T., et al. 2007, MNRAS, 380, 986

Caputi, K. I., Lagache, G., Yan, L., et al. 2007, ApJ, 660, 97

Chanial, P., Flores, H., Guiderdoni, B., et al. 2007, 462, 81

Calzetti, D., Armus, L., Bohlin, R. C., et al. 2000, 533, 682

Chapin, E. L., Hugues, D. H., Aretxaga, I., et al. 2009, MNRAS, 393, 653

Chary, R. R., \& Elbaz, D. 2001, ApJ, 556, 562

Chapman, S. C., Smail, I., Ivison, R. J., et al. 2002, ApJ, 573, 66

Chapman, S. C., Helou, G., Lewis, G. F., \& Dale, D. A. 2003, ApJ, 588, 186

Conroy, C. 2009, ApJ, 647, 201

Conroy, C., Gunn, J. E., \& White, M. 2009, ApJ, 699, 486

Cowie, L. L., \& Barger, A. J. 2008, ApJ, 686, 72

Cowie, L. L., Songaila, A., Hu, E. M., \& Cohen, J. G. 1996, AJ, 112, 839

Daddi, E., Dickinson, M., Morrison, G., et al. 2007, ApJ, 670, 172

Dale, D., \& Helou, G. 2002, ApJ, 576, 159

Dale, D. A., Helou, G., Contursi, A., Silbermann, N. A., \& Kolhatkar, S. 2001, ApJ, 549, 215

Damen, M., Labbé, I., Franx, M., et al. 2009, ApJ, 690, 937

da Cunha, E., Charlot, S., \& Elbaz, D. 2008, MNRAS, 388, 1595

Davé, R. 2008, MNRAS, 385, 147

Dickinson, M., Papovich, C., Ferguson, H. C., \& Budavári, T. 2003, ApJ, 587, 25

Drory, N., Bender, R., Feulner, G., et al. 2004, ApJ, 608, 742

Dunne, L., \& Eales, S. A. 2001, MNRAS, 327, 697

Dopita, M. A., Groves, B. A., Fischera, J., et al. 2005, ApJ, 619, 755

Dye, S. 2008, MNRAS, 328, 1293

Eales, S., Lilly, S., Gear, W., et al. 1999, ApJ, 515, 518

Elbaz, D., Daddi, E., Le Borgne, D., et al. 2007, A\&A, 468, 33

Erb, D. K., Steidel, C. C., Shapley, A. E., et al. 2006, ApJ, 646, 107

Fadda, D., Flores, H., Hasinger, G., et al. 2002, A\&A, 383, 838

Fazio, G. G., Hora, J. L., Allen, L. E., et al. 2004, ApJS, 154, 10

Feulner, G., Goronova, Y., Drory, N., Hopp, U., \& Bender, R. 2005, MNRAS, 358, L1

Fioc, M., \& Rocca-Volmerange, B. 1997, A\&A, 326, 950

Franceschini, A., Manners, J., Polletta, M. C., et al. 2005, AJ, 129, 2074

Fontana, A., Donnarumma, I., Vanzella, E., et al. 2003, ApJ, 594, 9

Genzel, R., Lutz, D., Sturm, E., et al. 1998, ApJ, 498, 579

Giacconi, R., Zirm, A., Wang, J. X., et al. 2002, ApJS, 139, 369

Gruppioni, C., Pozzi, F., Polletta., M., et al. 2008, ApJ, 684, 136

Iglesias-Páramo, J., Buat, V., Hernández-Fernández, J., et al. 2007, ApJ, 670, 279

Ishida, C. M. 2004, Thesis (Ph.D.), University of HAWAI'I, Source DAI-B 65/04, Oct., 1908, 502

Kauffmann, G., Heckman, T. M., White, S. D. M., et al. 2003, MNRAS, 341, 33 Kaviraj, S., Khochfar, S., Schawinski, K., et al. 2009, MNRAS, 394, 67 Kennicutt, R. C. 1998, ApJ, 498, 541 
Kennicutt, R. C., Jr., Lee, J. C., Funes, J. G., Sakai, S., \& Akiyama, S. 2005 , in Starbursts: From 30 Doradus to Lyman Break Galaxies, ed. R. de Grijs, \&

R. M. González Delgado (Dordrecht: Springer), ASSL, 329, 187

Kitzbichler, M. G., \& White, S. D. M. 2007, MNRAS, 376, 2

Lagache, G., Dole, H., \& Pujet, J.-L. 2003, MNRAS, 338, 555

Lagache, G., Dole, H., Pujet, J.-L., et al. 2004, ApJ, 154, 112

Lee, S.-K., Idzi, R., Ferguson, H. C., et al. 2009, ApJ, 184, 100

Le Borgne, D., Elbaz, D., Ocvirk, P., \& Pichon, C. 2009, A\&A, 504, 727

Le Floc'h, E., Papovich, C., Dole, H., et al. 2005, ApJ, 632, 169

Lewis, G. F., Chapman, S. C., Ibata, R. A., et al. 1998, ApJ, 505, L1

Magnelli, B., Elbaz, D., Chary, R. R., et al. 2009, A\&A, 496, 57

Manners, J. C., Serjeant, S., Bottinelli, S., et al. 2004, MNRAS, 355, 97

Maraston, C. 1998, MNRAS, 300, 872

Maraston, C. 2005, MNRAS, 362, 799

Maraston, C., Daddi, E., Renzini, A., et al. 2006, ApJ, 652, 85

Marchesini, D., van Dokkum, P. G., Förster Schreiber, N. M., et al. 2009, ApJ, 701,1765

Marcillac, D., Elbaz, D., Chary, R. R., et al. 2006, A\&A, 451, 57

Martin, C., Small, T., Schiminovich, D., et al. 2007, ApJS, 173, 415

Melbourne, J., Koo, D. C., \& Le Floc'h, E. 2005, ApJ, 632, L65

Noeske, K. G., Weiner, B. J., Faber, S. M., et al. 2007a, ApJ, 660, L43

Noeske, K. G., Weiner, B. J., Faber, S. M., et al. 2007b, ApJ, 660, L47

Noll, S., Pierini, D., Cimatti, E., et al. 2009a, A\&A, 499, 69

Noll, S., Burgarella., D., Giovannoli, E., et al. 2009b, A\&A, 507, 1793

Pannella, M., Hopp, U., Saglia, R. P., et al. 2006, ApJ, 639, 1

Pannella, M., Carilli, C. D., Daddi, E., et al. 2009, ApJ, 698, L116

Panuzzo, P., Silva, L., Granato, G. L., et al. 2005, AIP Conf. Proc., 761, 187

Papovich, C., Dole, H., Egami, E., et al. 2004, ApJS, 154, 70

Papovich, C., Moustakas, L. A., Dickinson, M., et al. 2006, ApJ, 640, 92

Pérez-González, P. G., Rieke, G. H., Egami, E., et al. 2005, ApJ, 630, 82

Reach, W. T., Dweck, E., Fixsen, D. J., et al. 1995, ApJ, 451, 188

Renzini, A. 2009, MNRAS, 398, L58

Rieke, G. H., Young, E. T., Engelbracht, C. W., et al. 2004, ApJS, 154, 25
Rieke, G. H., Alonso-Herrero, A., Weiner, B. J., et al. 2009, ApJ, 692, 556

Salim, S., Charlot, S., Rich, R. M., et al. 2005, ApJ, 619, L39

Salim, S., Rich, R., Charlot, S., et al. 2007, ApJS, 173, 267

Salim, S., Dickinson, M., Rich, R. M., et al. 2009, ApJ, 700, 161

Salimbeni, S., Fontana, A., Giallongo, E., et al. 2009, AIPC, 1111, 207

Sanders, D. B., \& Mirabel, I. F. 1996, A\&A, 34, 749

Santini, P., Fontana, A., Grazian, A., et al. 2009, A\&A, 504, 751

Seymour, N., Symeonidis, M., Page, M. J., et al. 2009, MNRAS, 398, 1573

Shapley, A. E., Steidel, C. C., Erb, D. K., et al. 2005, ApJ, 626, 698

Siebenmorgen, R., \& Krügel, E. 2007, A\&A, 461, 445

Siebenmorgen, R., Freudling, W., Krügel, E., \& Haas, M. 2004a, A\&A, 421, 129

Siebenmorgen, R., Krügel, E., \& Spoon, H. W. W. 2004b, A\&A, 414, 123

Silva, L., Granato, G. L., Bressan, A., \& Danese, L. 1998, ApJ, 509, 103

Silva, L., Maiolino, R., \& Granato, G. L. 2004, MNRAS, 355, 973

Soifer, B. T., \& Neugebauer, G. 1991, AJ, 101, 354

Stanford, S. A., Stern, D., Van Breugel, W., \& De Breuk, C. 2000, ApJS, 131, 185

Stern, D., Eisenhardt, P., Gorjian, V., et al. 2005, ApJ, 631, 163

Symeonidis, M., Page, M. J., Seymour, N., et al. 2009, MNRAS, 397, 1728

Taylor, E. N., Franx, M., van Dokkum, P. G., \& Quadri, R. F. 2009, ApJS, 183 295

Tonini, C., Maraston, C., Devriendt, J., Thomas, D., \& Silk, J. 2009, MNRAS, 396, 36

Valiante, E., Lutz, D., Sturm, E., Genzel, R., \& Chapin, E. L. 2009, ApJ, 701, 1814

Walcher, C. J., Lamareille, F., Arnouts, S., et al. 2008, A\&A, 491, 713

Wang, J. L., Xia, X. Y., Mao, S., et al. 2006, ApJ, 649, 722

Werner, M. W., Roellig, T. L., Low, F. J., et al. 2004, ApJS, 154,

Wolf, C., Meisenheimer, K., Kleinheinrich, M., et al. 2004, A\&A, 421, 913

Wuyts, S., Franx, M., Cox, T. J., et al. 2009, ApJ, 696, 348

Zheng, X. Z., Hammer, F., Flores, H., Assémat, F., \& Pelat, D. 2004, A\&A, 421, 847

Zheng, X. Z., Dole, H., Bell, E. F., et al. 2007, ApJ, 670, 301 